\title{
Assessing the utility of urinary and fecal cortisol as an indicator of stress in golden snub-nosed monkeys (Rhinopithecus roxellana)
}

\author{
Haochun Chen ${ }^{1,2}$, Hui Yao ${ }^{3}$, Wanji Yang ${ }^{1}$, Penglai Fan ${ }^{1}$, Zuofu Xiang ${ }^{\text {Corresp. } 1,2}$ \\ 1 Institute of Evolutionary Ecology and Conservation Biology, Central South University of Forestry \& Technology, Changsha, Hunan, China \\ 2 College of Life Science and Technology, Central South University of Forestry \& Technology, Changsha, Hunan, China \\ ${ }^{3}$ Key Lab of Conservation Biology for Shennongjia Golden Snub-nosed Monkeys, Hubei Province, Shennongjia Forest District, China \\ Corresponding Author: Zuofu Xiang \\ Email address: zorph@163.com
}

Cortisol concentration (CC) is often used as a stress indicator in animals, as high CC is associated with elevated stress levels. During field research, non-invasive methods of measuring $\mathrm{CC}$, such as collection of urine and feces, are superior to using blood samples when monitoring free-ranging animals' stress levels. However, due to different metabolic pathways, whether CC can be detected in urine and feces to reliably assess stress varies across species. Therefore, it is important to ascertain whether urine and fecal samples are a reliable source for determining CCs and to determine a suitable sampling regime. In this study, we subjected three captive adult golden snub-nosed monkeys (Rhinopithecus roxellana) to a high-stress situation (capture and injection). Urine and feces were collected for four days before and for four days after the manipulations for laboratory analysis. Immunoreactive CC was detected with a commercial enzyme immunoassay (EIA) kit and showed distinct rises. Peak CC values in urine were detected within 5 hours, while peak fecal CC ranged between 5 and 24 hours post-interference. These results provide evidence that $\mathrm{CC}$ in urine and feces can be used to assess stress levels in the golden snub-nosed monkey. The optimal time frame to collect urinary and fecal samples for CC analysis is within one day of a potential stressful event. 
1 Assessing the utility of urinary and fecal cortisol as an indicator of stress

2

3

4 Haochun Chen ${ }^{1,2}$, Hui Yao ${ }^{3}$, Wanji Yang ${ }^{1}$, Penglai Fan ${ }^{1}$, Zuofu Xiang ${ }^{1,2 *}$

51 Institute of Evolutionary Ecology and Conservation Biology, Central South University of Forestry \&

6 Technology, Changsha, Hunan, 410004, China

$7{ }^{2}$ College of Life Science and Technology, Central South University of Forestry \& Technology, Changsha,

8 Hunan, 410004, China

$9 \quad{ }^{3}$ Key Lab of Conservation Biology for Shennongjia Golden Snub-nosed Monkeys, Hubei Province, Shennongjia

Forest District, Hubei 442411, China

11

Zuofu Xiang (xiangzf@csuft.edu.cn; zorph@163.com)

Running head: Non-invasive stress monitoring in $R$. roxellana

\section{*Correspondence to:}

Zuo-Fu Xiang

498 ShaoshanNanlu, Changsha, Hunan, 410004, P. R. China

College of Life Science and Technology,

Central South University of Forestry \& Technology,

Tel: 86-731-5623392; Fax: 86-731-5623498 
22 Abstract: Cortisol concentration (CC) is often used as a stress indicator in animals, as high CC is associated

23 with elevated stress levels. During field research, non-invasive methods of measuring CC, such as using

24 urine or feces as hormone matrix, are usually beneficial compared to using blood sampling when monitoring

25 free-ranging animals' stress levels. However, due to different metabolic pathways, it is advisable to test

26 whether CC can be sufficiently detected in the urine or feces of a particular species to reliably assess stress.

27 In this study, we subjected three captive adult golden snub-nosed monkeys (Rhinopithecus roxellana) to a

high-stress situation (capture and injection). Urine and feces were collected for four days before and for

four days after the manipulation for hormone analysis. Immunoreactive cortisol was detected with a commercial enzyme immunoassay (EIA) kit and showed peak post-manipulation CC values in urine were detected within 5 hours, while a respective peak for fecal CC ranged between 5 and 24 hours postinterference. These results provide evidence that urinary and fecal $\mathrm{CC}$ can be used to assess glucocorticoid alteration in the golden snub-nosed monkey. The optimal time frame to collect urine or fecal samples for $\mathrm{CC}$ analysis is within one day of perception of a potential stressful event. 


\section{Introduction}

38 Cortisol, the primary glucocorticoid of primates, is released in response to stress (Fagot et al., 2014;

39 Whitten, Brockman \& Stavisky, 1998). Measuring cortisol levels in blood (serum/plasma) has proved to be

40 a useful indicator of perceived stress (Broom \& Johnson, 1993). Nonetheless, collecting blood from wild

animals is not easy, involving stressful procedures like restraint and sedation. Hence, non-invasive methods

such as collecting urine and feces to assess the stress response are becoming prevalent (Behie, Pavelka \&

Chapman, 2010; Möstl \& Palme, 2002; Novak et al., 2013; Whitten, Brockman \& Stavisky, 1998).

Although cortisol is the prominent biological active glucocorticoid in most primate species, only traces of

native cortisol may exist in the urine and feces of particular species, as it is usually converted into various

metabolites before being excreted (Hämäläinen et al., 2014; Möstl \& Palme, 2002). For instance, Bahr et

al. (2000) measured cortisol and several metabolites, and found that native cortisol was a major urinary

excretory product in common marmosets (Callithrix jacchus), while only small amounts were present in

the urine of long-tailed macaques (Macaca fascicularis) and chimpanzees (Pan troglodytes). Conversely,

fecal cortisol could barely be detected in common marmosets (Bahr et al., 2000). As a consequence, a

respective assay must be validated for any particular species and matrix to ensure proper quantification of cortisol or its metabolites (Sheriff et al., 2011).

Meanwhile, knowing the lag time between the secretion and excretion of glucocorticoids is beneficial

to better understand the connection between physiological alterations and behavioral events/states, but the 
contrast, the lag time for the appearance of cortisol in feces is both longer and more variable among species, since defecation frequencies are affected by diet and gut passage time. Although not always the case (Goymann, 2012; Hämäläinen et al., 2014), hormones are more slowly excreted in the feces of largerbodied primate species. Hormones excreted in feces showed a delay of $44 \mathrm{~h}$ in Western lowland gorillas (Gorilla gorilla gorilla) (Shutt, Setchell \& Heistermann, 2012), 26 h in olive baboons (P. cynocephalus), $22 \mathrm{~h}$ in chimpanzees (Wasser et al., 2000), and 8-16 h in squirrel monkeys (Saimiri sciureus) (Moorman et al., 2002). Thus, for each species, it is also important to identify the lag time for cortisol excretion (or its metabolites) besides validating the method to monitor the stress response.

The golden snub-nosed monkey (Rhinopithecus roxellana) is an Asian colobine endemic to China. Its discontinuous geographic distribution occurs in the provinces of Sichuan, Gansu, Shaanxi, and Hubei (Li, Pan \& Oxnard, 2002). It is listed as Endangered by the International Union for Conservation of Nature (IUCN, 2014). Golden snub-nosed monkeys live in a multilevel or modular society, in which several one-male and multi-female units with one or several all-male units form a band that feeds, forages, travels and rests together (Zhang et al., 2006). Bachelor males in all-male units have to fight for dominance, while the resident males in one-male units face mating competition and risk of being deposed by the bachelor males. Furthermore, primates living in the wild routinely experience stressful situations including dominance interactions, diseases, parasitism, predation, and food shortages (Novak et al., 2013). With the development of ecotourism, golden snub-nosed monkeys may also experience stress from close proximity to humans (Maréchal et al., 2011; Xiang et al., 2011). Hence, the possibility to monitor the physiological state of golden snub-nosed monkeys would be valuable option to evaluate their health and well-being. However, no published studies have validated whether urinary or fecal cortisol or its metabolites can be used as 
indicators of perceived stress in $R$. roxellana. In this experiment, by handling three golden snub-nosed monkeys and injecting a saline solution we stimulated a potential stress response, which was subsequently measured by enzyme immunoassay (EIA). Our aims were to validate an EIA for monitoring urinary and fecal cortisol concentrations (CC), and to determine the lag time of urinary and fecal CC for captive golden snub-nosed monkeys.

\section{Methods}

\section{Ethics Statement}

Prior to conducting this study, approval was gained from the Shennongjia National Nature Reserve (snnr-081201), and the Institutional Animal Care and Use Committee of Central South University of Forestry \& Technology (csuft-090120).

\section{Animals and housing}

The animals used in this study had been rescued from illness or injury and were being reared in cages, as they were not yet ready for reintroduction into the wild. Three adult golden snub-nosed monkeys, two males (QQ and TT) and one female (SN), were chose as subjects at Xiaolongtan conservation station, Shennongjia National Park (SNP), Hubei, China. QQ was housed with two females and an infant. TT was housed with SN. Of the three, only SN was captive-born at Xiaolongtan. Each enclosure was $25 \mathrm{~m}^{2}$ in area, $5 \mathrm{~m}$ high, and contained a dead tree fixed in the middle. There was a small cage connected to each enclosure for resting and sleeping. The enclosures were built 20-30 centimeters above a cement foundation. Rails were 3-6 meters away from the enclosures to keep tourists away from the animals. The animals were consistently fed peaches, apples, and other similar foods three times a day throughout the experiment. Water was available ad libitum. 
100

101

102

103

104

105

106

107

108

109

110

111

112

113

114

115

116

117

118

119

120

\section{Validation Experiment}

We imposed a potentially acute stressor (capture and injection) on the three focal animals by entering their cages with several reserve employees, trapping the monkeys with large bags, and thus restraining them before giving each one a saline intramuscular injection. The interventions were conducted in sequence, and we could not stop the monkeys from watching the capture of other monkeys. Each capture took less than 15 minutes, and the entire procedure was conducted within an hour. Due to the acute stress, the monkeys breathed heavily and defecated once or twice. However, after injection, subjects appeared to recover in minutes, and aside from mild diarrhea in QQ no abnormal behavior was recorded that afternoon. Prior to this intervention, all subjects had been habituated to the presence of investigators and the collection procedure for urine and fecal samples for 20 days, and no investigator took part in the capture to reduce the potential of additional perceived stress during the post-intervention period. The monkeys had never experienced this intervention procedure prior to this study.

\section{Sample collection and storage}

Samples were obtained during the day for 4 days before and 4 days after the stress manipulation. Two investigators stood alongside each enclosure for sampling from 7:00-12:00 and again from 13:00-18:00. Samples were always collected from outside of the enclosure to avoid potential disturbance of the monkeys; as they usually rested and defecated near the edges of the cage. We collected no more than $4 / 3$ urine/fecal samples for each individual per day, but during the first two days post-injection, we collected every available sample with help of colleagues except for overnight and early morning defecation (before 7: 00), which resulted in a total of 31 urine samples for QQ (13 urine samples pre-intervention, and 18 urine samples post-intervention), 47 for TT (15 urine samples pre-intervention, and 32 urine samples post- 
121

122

123

124

125

126

127

128

129

130

131

132

133

134

135

136

137

138

139

140

141

intervention), and 30 for SN（16 urine samples pre-intervention, and 14 urine samples post-intervention ）;

as well as 31 fecal samples for QQ (10 fecal samples pre-intervention, and 21 fecal samples postintervention) and 16 for TT ( 7 fecal samples pre-intervention, and 9 fecal samples post-intervention). The collected number of fecal samples for SN was insufficient ( $n=9)$, especially as no fecal sample of SN was collected in the first 30 hours post injection, so we excluded this sample set from all subsequent analyses.

Fresh feces were collected from the clean, dry cage floor using steel clamps and immediately placed in a Ziploc bag. The clamps were washed and dried after every collection. A minimum of $0.5 \mathrm{~mL}$ of urine were collected in a disposable plastic bag attached to a long stick (Fig. 1). Holding the stick, we collected the monkeys' urine in the bag, thereafter transferring the urine into centrifuge tubes by syringe. If the bag was not placed in time to catch falling urine, urine on the floor was collected using a syringe if it was in reach of the investigators. Urine and feces were discarded in the event of cross-contamination with each other or with water. All samples were collected and analyzed separately. Once collected, samples were stored in a portable ice box filled with ice bags until they could be placed in the freezer $\left(-20^{\circ} \mathrm{C}\right)$ within four hours of collection. Samples were kept frozen until hormone analyses were performed at Central South University of Forestry and Technology.

\section{Fig. 1}

\section{Pre-treatment of samples}

Urine samples were centrifuged at $4000 \mathrm{rpm}$ for $15 \mathrm{~min}$ after thawing at ambient temperature. Then the supernatant was diluted with assay buffer 400 times prior to cortisol assay and 20 times prior to creatinine assay, in order to make the results fall within the range of the respective standard curves (dilution ratios were determined previously using pilot assays). 
142

143

144

145

146

147

148

149

150

151

152

153

154

155

156

157

158

159

160

161

162

Fecal samples were processed based on the method described by Wasser et al. (2000), and Fan et al.

(2013), and the instructions provided by the EIA kit manufacturer. Fully lyophilized, powdered fecal

samples $(0.1 \mathrm{~g})$ were put into $1.5 \mathrm{~mL}$ centrifuge tubes containing $1 \mathrm{~mL}$ of ethanol (100\%). After 30 minutes

of shaking, samples were centrifuged at $4000 \mathrm{rpm}$ for $15 \mathrm{~min}$. The supernatant was transferred into a clean

tube, then evaporated to dryness in a $60^{\circ} \mathrm{C}$ water bath. Extracted samples were re-dissolved with $100 \mu \mathrm{L}$

ethanol, followed by $900 \mu \mathrm{L}$ of Assay Buffer (AB, PBS added bull serum albumin). A volume of $100 \mu \mathrm{L}$

was taken out and diluted with $200 \mu \mathrm{L}$ AB prepared for assay.

\section{Enzyme immunoassay (EIA)}

Cortisol concentration was assessed with a commercial EIA kit (catalogue \#K003-H5) from Arbor Assays (Ann Arbor, USA). According to the manufacturer, cross reactivity of the cortisol antibody is $100 \%$ for cortisol, $18.8 \%$ for dexamethasone, $7.8 \%$ for prednisolone, $1.2 \%$ for both corticosterone and cortisone, and less than $0.1 \%$ for progesterone. Intra-assay coefficients of variation are $6.5 \%(n=5)$ and $7.8 \%(n=5)$

for high- and low-concentration quality controls. Inter-assay coefficients of variation are $9.3 \%(\mathrm{n}=5)$ and $10.2 \%(n=5)$ for high- and low-concentrated quality controls. Assay protocols were based on the product instructions, except that standards were at $3200,1600,800,400,200$, and $100 \mathrm{pg} / \mathrm{mL}$ in urine cortisol assays, but were halved in assays for fecal samples to get better results. Optical densities were read at 450 nm with a plate reader (DNM 9602, Pulang, Beijing). Cortisol levels were calculated using an online fourparameter logistic curve-fitting program the manufacturer provided.

To determine the degree of parallelism for the EIA, a fecal extract pool and a urine pool were serially diluted in AB buffer, assayed, and compared with the respective standard curve. Results were plotted as the percentage bound vs. the log concentration measured. 
Assay accuracy was assessed for urine and feces respectively. A urine/feces pool containing low CC

164

165

166

167

168

169

170

171

172

173

174

175

176

177

178

179

180

181

182

183

was mixed with another urine/feces pool containing high CC (the $\mathrm{CC}$ was determined in pilot assays) in different ratios $(2: 8,4: 6,6: 4$, and 8:2) and subsequently analyzed. Regression curves of measured and expected cortisol concentrations are presented in Figure 2.

\section{Determination of creatinine}

To adjust for variations in water content, urinary $\mathrm{CC}$ was indexed against creatinine and expressed as $\mu \mathrm{g} / \mathrm{mg}$ Cr. Creatinine level was determined by a urinary creatinine detection kit from Arbor Assays (catalogue \#K002-H5) based on the Jaffe reaction (Taussky, 1954). The optical density was read at $490 \mathrm{~nm}$ with a plate reader (DNM 9602, Pulang, Beijing). Creatinine levels were calculated using an online fourparameter logistic curve-fitting program the manufacturer provided

\section{Statistical analysis}

The degree of parallelism of serial dilutions of steroid extracts to the standard curve were assessed with an ANCOVA for testing whether sample pool curves were similar to a standard curve, comparing slopes and intercepts respectively. Assay accuracy was assessed by mean percent recovery. Percent recovery was calculated based on measured CC dividing by expected CC. A mean percent recovery between $90 \%$ and $110 \%$ was determined to be an acceptable degree of accuracy.

Lag time was defined as the time between the stress manipulation and the occurrence of the highest individual signal of immunoreactive $\mathrm{CC}$ post-manipulation. The time at which the injections were conducted was designated as Time $=0$. Individual baseline $\mathrm{CCs}$ were expressed as median $( \pm$ the interquartile range). The Mann-Whitney U-test with Bonferonni correction was used to compare individual differences in baseline CCs. We eliminated the urine samples collected the same day after the injection and 
184 the fecal samples collected for two days after the injection when calculating the medians and performing 185 the Mann-Whitney test.

Data were processed in Microsoft Excel 2010 and SPSS 19.0. Two-tailed significance levels were set

187

188

189

190

191

192

193

194

195

196

197

198

199

200

201

202

203

204

at $p=0.05$.

\section{Results}

Serial dilutions of pool samples yielded similar curves to the standard cortisol curve (Fig 2A, for urine:

slope, $F_{1,6}=0.02, p=0.964$; intercept, $\mathrm{F}_{1,7}=1.075, p=0.334$; for feces: slope, $\mathrm{F}_{1,6}=2.073, p=0.204$; intercept, $\left.\mathrm{F}_{1,7}=0.028, p=0.871\right)$. In the accuracy test, the values of $\mathrm{r}^{2}$ were 0.9900 for urine samples and 0.9949 for fecal samples. Mean percent recovery was $100.8 \%(n=4)$ for urine samples and $96.3 \%$ for fecal samples $(n=4)$.

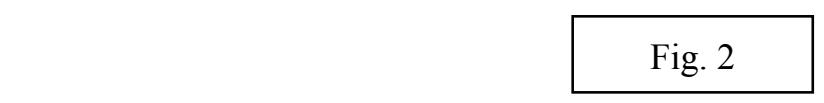

The individual urinary baseline CCs of TT, QQ, and SN were $1.39( \pm 0.69) \mu \mathrm{g} / \mathrm{mg} \mathrm{Cr}(\mathrm{n}=39), 0.54( \pm$ $0.60) \mu \mathrm{g} / \mathrm{mg} \mathrm{Cr}(\mathrm{n}=26)$, and $0.47( \pm 0.57) \mu \mathrm{g} / \mathrm{mg} \mathrm{Cr}(\mathrm{n}=29)$, respectively. The post-stressor peak urinary CCs increased about 10-fold (13.18 $\mu \mathrm{g} / \mathrm{mgCr})$ for TT, 5-fold $(2.62 \mu \mathrm{g} / \mathrm{mg} \mathrm{Cr})$ for QQ, and 11-fold (5.03 $\mu \mathrm{g} / \mathrm{mg} \mathrm{Cr}$ ) for SN above their individual baseline levels. The fecal cortisol baseline levels of TT and QQ were $30.95( \pm 8.20) \mathrm{ng} / \mathrm{g}$ feces $(\mathrm{n}=11)$ and $14.87( \pm 16.23) \mathrm{ng} / \mathrm{g}$ feces $(\mathrm{n}=22)$ respectively, and peak fecal CCs increased about 3-fold (80.28 ng/g feces) for TT and 6-fold (86.16 ng/g feces) for QQ. TT had significantly higher baseline urinary and fecal cortisol levels compared to QQ (urine, $U_{39,26}=74, p<0.001$; feces, $\left.U_{11,22}=43, p<0.05\right)$, and significantly higher baseline urinary cortisol levels than $\mathrm{SN}\left(U_{39,29}=120\right.$, $p<0.001)$. No significant difference was found between QQ and SN's baseline urinary cortisol levels $\left(U_{26}\right.$, $\left.{ }_{29}=352, p>0.05\right)$. 
205

206

207

208

209

210

211

212

213

214

215

216

217

218

219

220

221

222

223

224

225

Peak immunoreactive $\mathrm{CCs}$ in urine appeared $3.5 \mathrm{~h}(\mathrm{n}=3, \mathrm{SD}=1.6)$ post-injection (Fig 3). Peak immunoreactive CCs in feces were detected at $5 \mathrm{~h}$ in TT, and at $22.9 \mathrm{~h}$ in QQ (Fig 3).

Fig. 3

\section{Discussions}

Based on the responses of three captive adult golden snub-nosed monkeys in a high-stress situation (capture and injection), our results clearly demonstrate the suitability of the EIA to reliably detect alterations in immunoreactive $\mathrm{CC}$ in urine and feces of golden snub-nosed monkeys.

The average lag time for urinary peak CC was $3.5 \mathrm{~h}(\mathrm{n}=3)$ for this study, which is consistent with results from earlier studies (Bahr et al., 2000; Smith \& French, 1997), usually indicating lag times for a respective signal in urine of 2-6 hours. The lag time for fecal cortisol peak CCs in the present study were 5 and 23 hours. This result is similar to findings for chimpanzees, olive baboons, and long-tailed macaques, demonstrating a respective lag time for urinary glucocorticoid output between 8 and 26 hours (Bahr et al., 2000; Wasser et al., 2000). However, we believe that for golden snub-nosed monkeys the usual lag time for fecal clearance is closer to 23 hours than it is to the 5-hour result for three reasons. First, TT, on an individual level, might have been more susceptible to the imposed stressor than QQ or SN, potentially leading an increased metabolic rate or gastro-intestinal motility, resulting in quicker hormone excretion through feces (Goymann, 2012; Steinbrook, 1998). Secondly, we might have missed the actual peak sample if it was voided overnight or in the early hours of day post-intervention. Thirdly, TT had urinary and fecal baseline

CCs more than twice as high as those of QQ, and urinary baseline CCs nearly three times higher than the female monkey SN. The comparatively higher baseline CCs of TT indicate that TT might perceive more 
226

227

228

229

230

231

232

233

234

235

236

237

238

239

240

241

242

243

244

245

246

stress than the other two individuals, although we can't exclude the possibility of an individual difference

in baseline levels. However, both lag time and baseline level of urinary CCs are comparable between QQ

and SN, which might indicate neglectable differences in potentially existing sex-related differences in steroid metabolism.

In conclusion, we have validated a reliable EIA method to monitor CC which can be used in future

study. Cortisol lag time is $3.5 \mathrm{~h}$ in urine, and $23 \mathrm{~h}$ in feces, meaning corresponding $\mathrm{CC}$ changes to certain stressor would show shortly in urine, but probably in the next day in feces. So we recommend to use urine sample if it is possible.

\section{Acknowledgements}

We thank the Administration Bureau of Shennongjia National Park for their support in field work. We thank Bo Zhang, XuejunLuo, Hanlong Chen, Ruoshuang Liu for collecting samples.

\section{References}

Bahr NI, Palme R, Mohle U, Hodges JK, Heistermann M. 2000. Comparative aspects of the metabolism and excretion of cortisol in three individual nonhuman primates. General and Comparative Endocrinology 117(3):427-38. DOI:10.1006/gcen.1999.7431.

Behie AM, Pavelka MSM, Chapman CA. 2010. Sources of variation in fecal cortisol levels in howler monkeys in Belize. American Journal of Primatology 72(7): 600-606. DOI:10.1002/ajp.20813.

Broom DM, Johnson KG. 1993. Stress and animal welfare. Springer Science \& Business Media. DOI: 10.1007/978-94-024-0980-2. 
247 Fan PL, Chen HC, Yao H, Wang ZL, Yang JY, Xiang ZF. 2013. Measurement of urinary and fecal steroid

248

249

250

251

252

253

254

255

256

257

258

259

260

261

262

263

264

265

266

267

metabolites in a provisioned group of golden snub-nosed monkeys (Rhinopithecus roxellana) at Shennonjia Reserve, Hubei, China (in Chinese). Acta Theriologica Sinica 33(3): 286-292.

Fagot J, Gullstrand J, Kemp C, Defilles C, Mekaouche M. 2014. Effects of freely accessible computerized test systems on the spontaneous behaviors and stress level of Guinea baboons (Papio papio). American Journal of Primatology 76(1): 56-64. DOI: 10.1002/ajp.22193.

Goymann W. 2012. On the use of non-invasive hormone research in uncontrolled, natural environments: the problem with sex, diet, metabolic rate and the individual. Methods in Ecology and Evolution 3(4):757-765. DOI: 10.1111/j.2041-210X.2012.00203.x.

Hämäläinen A, Heistermann M, Fenosoa ZS, Kraus C. 2014. Evaluating capture stress in wild gray mouse lemurs via repeated fecal sampling: method validation and the influence of prior experience and handling protocols on stress responses. General and Comparative Endocrinology 195:68-79. DOI:10.1016/j.ygcen.2013.10.017.

IUCN 2014.The IUCN Red List of Threatened Species. Version 2014.3.<www.iucnredlist.org>. Downloaded on 29 December 2014.

Li BG, Pan RL, Oxnard CE. 2002. Extinction of snub-nosed monkeys in China during the past 400 years. International Journal of Primatology 23(6):1227-1244. DOI: 10.1023/A:1021122819845.

Möstl E, Palme R. 2002. Hormones as indicators of stress. Domestic Animal Endocrinology 23(1):67-74. DOI: 10.1016/S0739-7240(02)00146-7.

Maréchal L, Semple S, Majolo B, Qarro M, Heistermann M, MacLarnon A. 2011. Impacts of tourism on anxiety and physiological stress levels in wild male Barbary macaques. Biological Conservation 
144(9):2188-2193. DOI: 10.1016/j.biocon.2011.05.010.

Moorman EA, Mendoza SP, Shideler SE, Lasley BL. 2002. Excretion and measurement of estradiol and progesterone metabolites in the feces and urine of female squirrel monkeys (Saimiri sciureus). American Journal of Primatology 57:79-90. DOI: 10.1002/ajp.10036.

Novak MA, Hamel AF, Kelly BJ, Dettmer AM, Meyer JS. 2013. Stress, the HPA axis, and nonhuman primate well-being: A review. Applied Animal Behaviour Science 143(2-4): 135-149. DOI: 10.1016/j.applanim.2012.10.012.

Sheriff MJ, Dantzer B, Delehanty B, Palme R, Boonstra R. 2011. Measuring stress in wildlife: techniques for quantifying glucocorticoids. Oecologia 166(4), 869-887. DOI: 10.1007/s00442-011-1943-y.

Shutt K, Setchell JM, Heistermann M. 2012. Non-invasive monitoring of physiological stress in the Western lowland gorilla (Gorilla gorilla gorilla): Validation of a fecal glucocorticoid assay and methods for practical application in the field. General and Comparative Endocrinology 179(2):167177. DOI: $10.1016 /$ j.ygcen.2012.08.008.

Smith TE, French JA. 1997. Psychosocial stress and urinary cortisol excretion in marmoset monkeys (Callithrix kuhli). Physiology \& Behavior 62(2):225-232. DOI: 10.1016/S0031-9384(97)00103-0.

Steinbrook RA. 1998. Epidural anesthesia and gastrointestinal motility. Anesthesia \& Analgesia 86(4):837844. DOI: 10.1097/00000539-199804000-00029.

Taussky HH. 1954. A microcolormetric determination of creatinine in urine by the Jaffe reaction. The Journal of Biological Chemistry 208:853-861.

Wasser SK, Monfort SL, Southers J, Wildt DE. 1994. Excretion rates and metabolites of oestradiol and progesterone in baboon (Papio cynocephalus cynocephalus) faces. Journal of Reproduction and 
289

290

291

292

293

294

295

296

297

298

299

300

301

302

303

304

305

306

307

308

309

Fertility 101(1):213-20. DOI: 10.1530/jrf.0.1010213.

Wasser SK, Hunt KE, Brown JL, Cooper K, Crockett CM, Bechert U, Millspaugh JJ, Larson S, Monfort

SL. 2000. A generalized fecal glucocorticoid assay for use in a diverse array of nondomestic mammalian and avian species. General and Comparative Endocrinology 120(3):260-75. DOI: 10.1006/gcen.2000.7557.

Whitten P, Brockman D, Stavisky R. 1998. Recent advances in noninvasive techniques to monitor hormone-behavior interactions. American Journal of Physical Anthropology 107(S27):1-23. DOI: 10.1002/(SICI)1096-8644(1998)107:27+<1::AID-AJPA2>3.0.CO;

2-H.

Xiang ZF, Yu Y, Yang M, Yang JY, Liao MY, Li M. 2011. Does flagship species tourism benefit conservation? A case study of the golden snub-nosed monkey in Shennongjia National Nature Reserve. Chinese Science Bulletin 56(24):2553-2558. DOI: 10.1007/s11434-011-4613-x.

Zhang P, Watanabe K, Li B, Tan LC. 2006. Social organization of Sichuan snub-nosed monkeys (Rhinopithecus roxellana) in the Qinling Mountains, Central China. Primates 47(4):374-82. DOI: 10.1007/s10329-006-0178-8.

\section{Figures}

Fig. 1 Urine sampling device. 
310 Fig. 2 Validation results for detecting urinary and fecal cortisol in the golden snub-nosed monkey

311 (Rhinopithecus roxellana). A: Parallism test; the B/B0\% were calculated by optical densities of

312 standard/samples comparing to optical densities of blanks then multiplied by $100 \%$.B: Accuracy test.

313

314 Fig. 3 Longitudinal profile of urinary and fecal cortisol concentrations for three golden snub-nosed

315 monkeys (Rhinopithecus roxellana) (TT, QQ, and SN) following stress manipulation. (A, TT; B,QQ; C, $316 \mathrm{SN})$ 


\section{Figure 1}

Figure 1

Urine sampling device. 


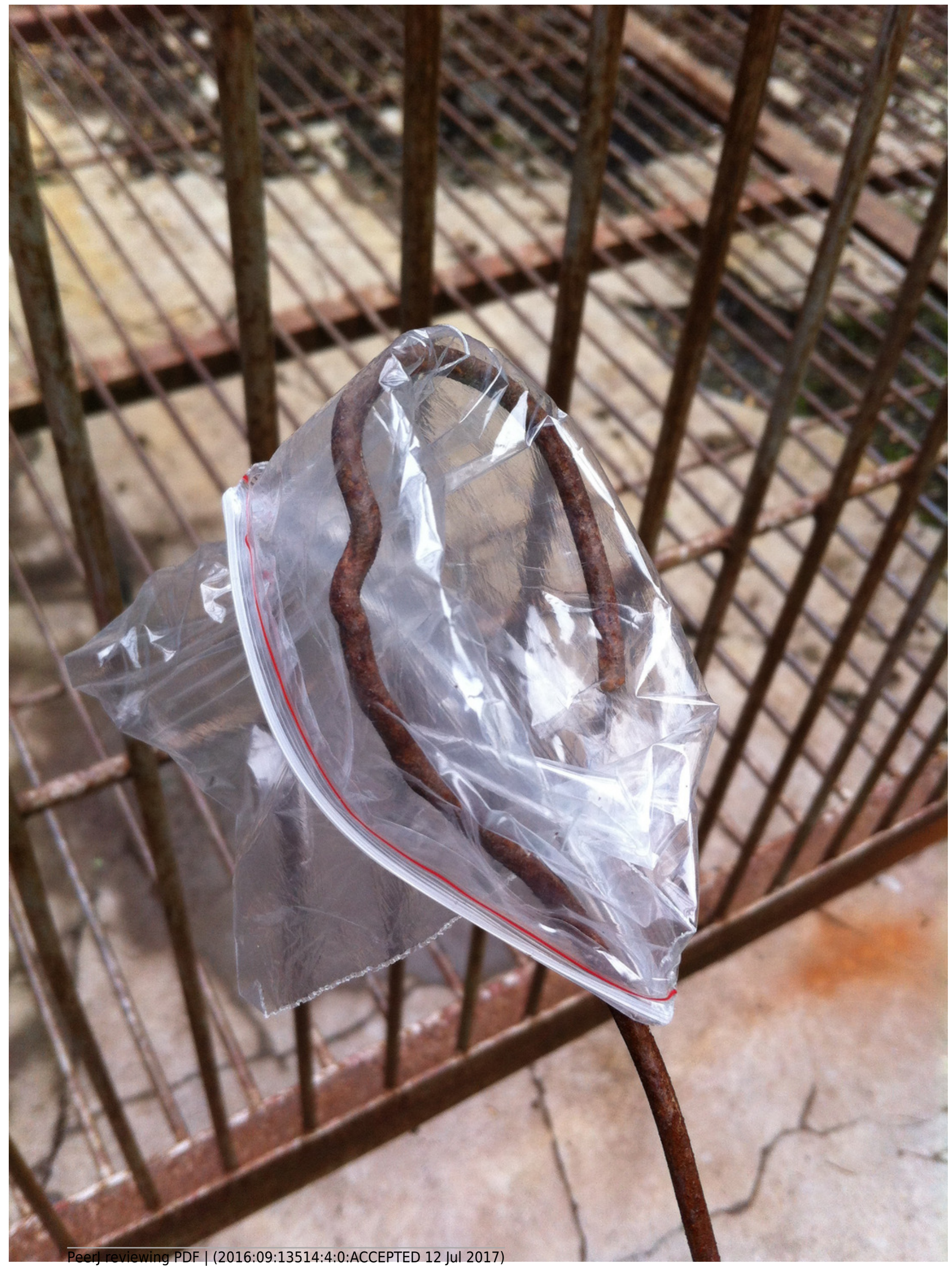


Figure 2

Figure 2

Validation results for detecting urinary and fecal cortisol in the golden snub-nosed monkey (Rhinopithecus roxellana). A: Parallism test; the B/B0\% were calculated by optical densities of standard/samples comparing to optical densities of blanks then multiplied by $100 \% . B$ :

Accuracy test.
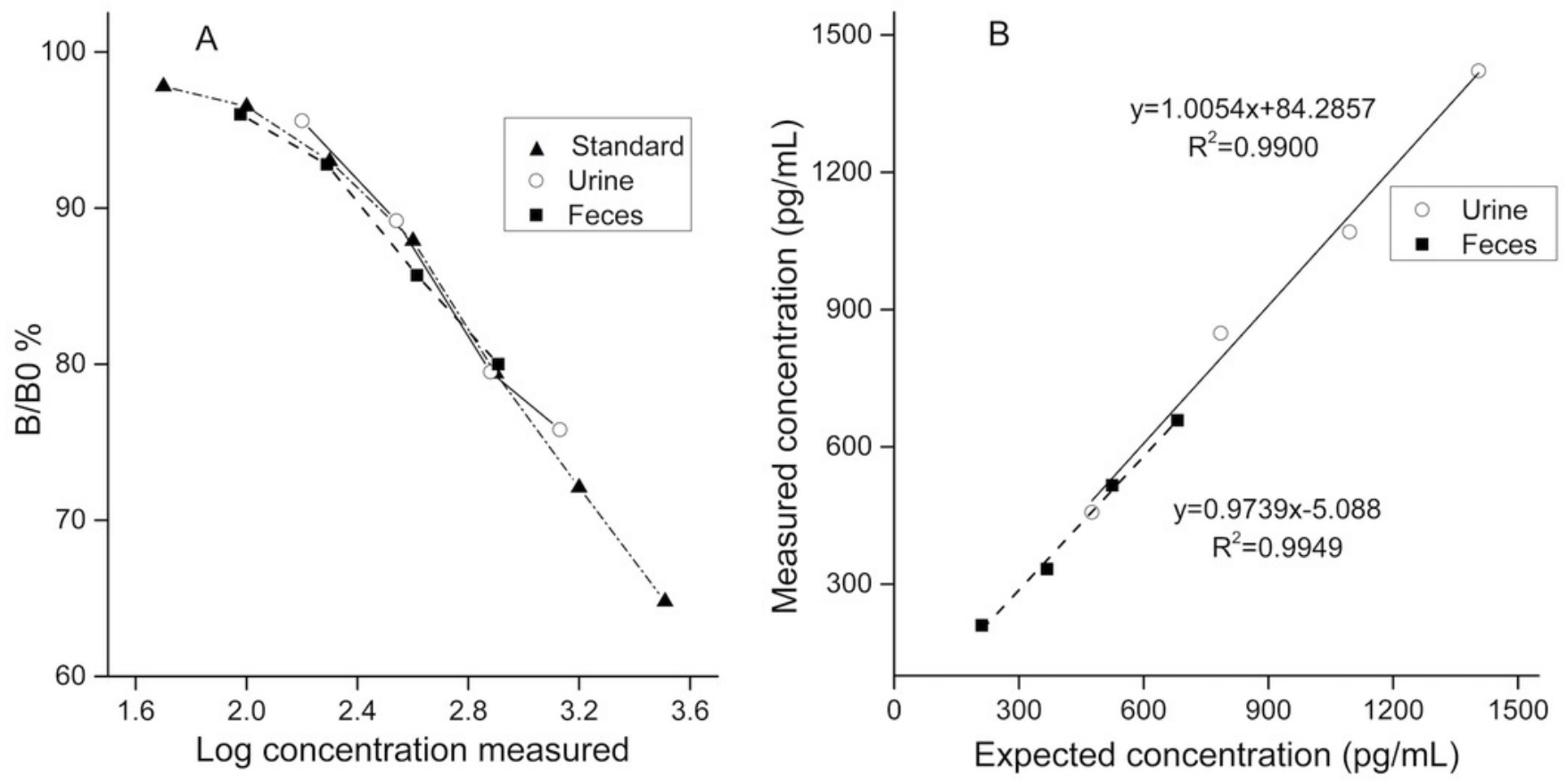


\section{Figure 3}

Figure 3

Longitudinal profile of urinary and fecal cortisol concentrations for three golden snub-nosed monkeys (Rhinopithecus roxellana) ( $\mathrm{T}, \mathrm{QQ}$, and $\mathrm{SN}$ ) following stress manipulation. (A, $\mathrm{TT}$; $B, Q Q ; C, S N)$. 


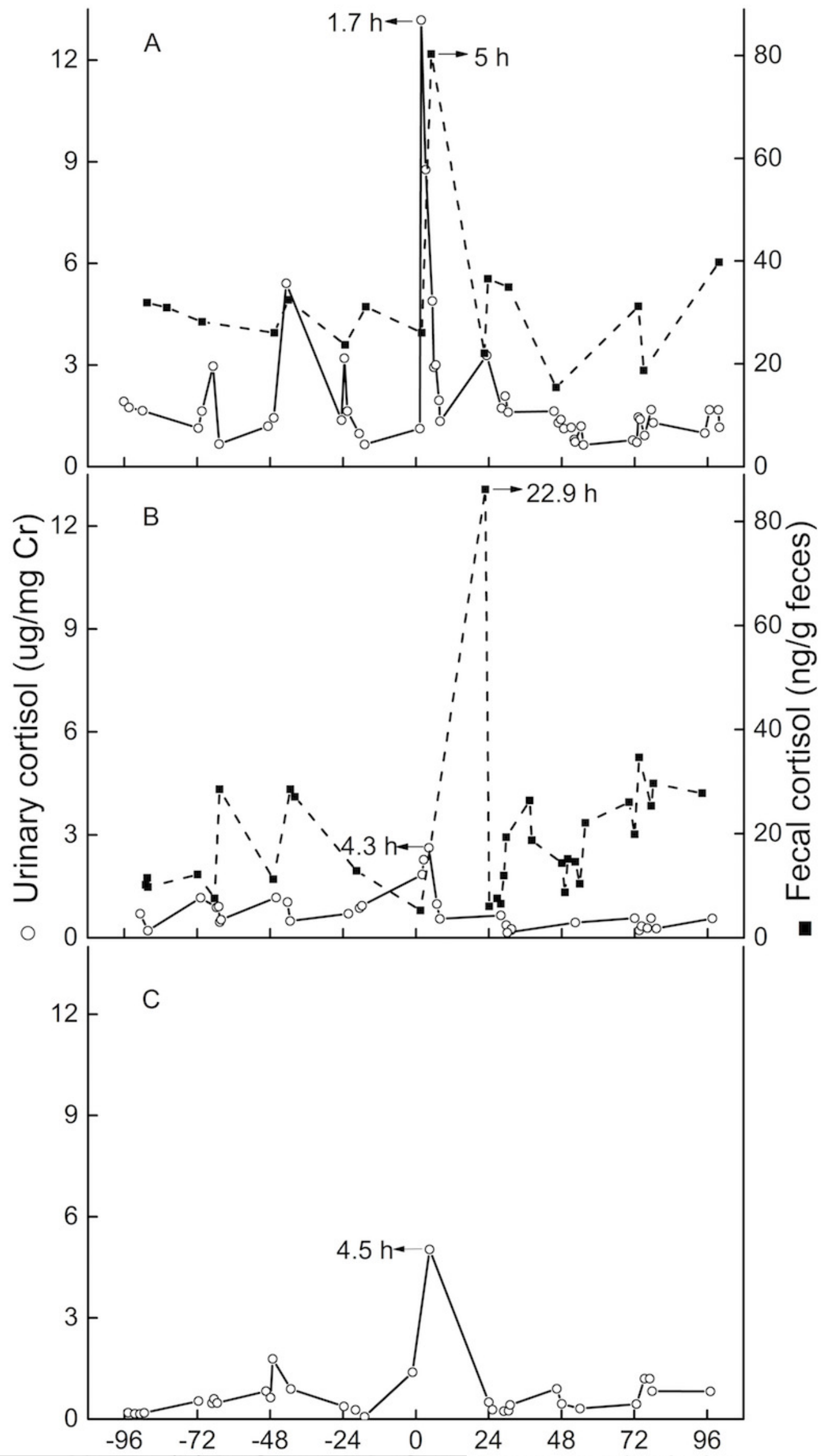

